\title{
Anterolateral Entorhinal Cortex Volume Predicted by Altered Intra-Item Configural Processing
}

\author{
ㄴok-Kin Yeung, ${ }^{1}$-Rosanna K. Olsen, ${ }^{2}$ Hannah E.P. Bild-Enkin, ${ }^{1}$ Maria C. D’Angelo, ${ }^{2}{ }^{\top}$ Arber Kacollja, ${ }^{2}$ \\ Douglas A. McQuiggan, ${ }^{2}$ Anna Keshabyan, ${ }^{1}$ Jennifer D. Ryan, ${ }^{1,2,3}$ and ${ }^{\circ}$ Morgan D. Barense ${ }^{1,2}$ \\ ${ }^{1}$ Department of Psychology, University of Toronto, Toronto, Ontario M5S 3G3, Canada, ${ }^{2}$ Rotman Research Institute, Baycrest Health Sciences, Toronto, \\ Ontario M6A 2E1, Canada, and ${ }^{3}$ Department of Psychiatry, University of Toronto, Toronto, Ontario M5T 1R8, Canada
}

Recent functional imaging studies have proposed that the human entorhinal cortex (ERC) is subdivided into functionally distinct anterolateral (alERC) and posteromedial (pmERC) subregions. The alERC overlaps with regions that are affected earliest by Alzheimer's disease pathology, yet its cognitive function remains poorly understood. Previous human fMRI studies have focused on its role in object memory, but rodent studies on the putatively homologous lateral entorhinal cortex suggest that it also plays an important role in representing spatial properties of objects. To investigate the cognitive effects of human alERC volume differences, we developed an eye-tracking-based task to evaluate intra-item configural processing (i.e., processing the arrangement of an object's features) and used manual segmentation based on a recently developed protocol to delineate the alERC/pmERC and other medial temporal lobe (MTL) subregions. In a group of older adult men and women at varying stages of brain atrophy and cognitive decline, we found that intra-item configural processing, regardless of an object's novelty, was strongly predicted by alERC volume, but not by the volume of any other MTL subregion. These results provide the first evidence that the human alERC plays a role in supporting a distinct aspect of object processing, namely attending to the arrangement of an object's component features.

Key words: anterolateral entorhinal cortex; configural processing; eye tracking; medial temporal lobe; mild cognitive impairment; MRI volumetry

\section{Significance Statement}

Alzheimer's disease pathology appears earliest in brain regions that overlap with the anterolateral entorhinal cortex (alERC). However, the cognitive role of the alERC is poorly understood. Previous human studies treat the alERC as an extension of the neighboring perirhinal cortex, supporting object memory. Animal studies suggest that the alERC may support the spatial properties of objects. In a group of older adult humans at the earliest stages of cognitive decline, we show here that alERC volume selectively predicted configural processing (attention to the spatial arrangement of an object's parts). This is the first study to demonstrate a cognitive role related to alERC volume in humans. This task can be adapted to serve as an early detection method for Alzheimer's disease pathology.

\section{Introduction}

Recent studies have highlighted the division of the human entorhinal cortex (ERC) into anterolateral (alERC) and posteromedial

\footnotetext{
Received Nov. 28, 2016; revised April 20, 2017; accepted April 25, 2017.

Author contributions: L.-K.Y., R.K.O., J.D.R., and M.D.B. designed research; L.-K.Y., R.K.O., H.E.P.B.-E., M.C.D., A. Kacollja, D.A.M., and A. Keshabyan performed research; R.K.O. contributed unpublished reagents/analytic tools; L.-K.Y., J.D.R., and M.D.B. analyzed data; L.-K.Y., R.K.O., J.D.R., and M.D.B. wrote the paper.

This work was supported by the Canadian Natural Sciences Engineering Research Council (Discovery and Accelerator Grants to M.D.B.; (anada Graduate Scholarship, Doctoral Program, to L.-K.Y.), the James S. McDonnell Foundation (Scholar Award to M.D.B.), and the Canada Research Chairs Program (J.D.Rand M.D.B.). We thank Dr. Nicholas Rule for assistance with multiple regression analysis.

The authors declare no competing financial interests.

L.-K. Yeung's present address: Taub Institute, Columbia University Medical Center, New York, NY 10032.

Correspondence should be addressed to Lok-Kin Yeung, Taub Institute, Columbia University Medical Center, 630

West 168th Street, P.0. Box 16, New York, NY 10032. E-mail: Iy2143@cumc.columbia.edu.
}

(pmERC) halves, which belong to functionally distinct pathways and support different cognitive roles (Maass et al., 2015; Navarro Schröder et al., 2015). Critically, the alERC overlaps significantly with the regions described as transentorhinal cortex (Braak and Braak, 1991) or lateral entorhinal cortex (Khan et al., 2014), which exhibit Alzheimer's disease pathology earlier than any other brain region. Despite the clinical importance of alERC decline, no human studies to date have attempted to link alERC volume to particular cognitive functions.

The alERC and pmERC are thought to be the human homologs of the rodent lateral (LEC) and medial (MEC) entorhinal 
cortices, which belong to two distinct functional pathways in the rodent brain. Neuroanatomical studies show that the LEC primarily receives projections from the perirhinal cortex (PRC), whereas the MEC receives most of its input from the postrhinal cortex, the rodent analog of the human parahippocampal cortex (PHC) (Naber et al., 1997; see also Suzuki and Amaral, 1994 for the same anatomical dissociation in monkeys). In turn, the LEC and MEC project to distinct regions of CA1 and the subiculum in the hippocampus (Witter, 1993). However, the separation between these two pathways is not absolute; for example, there are reciprocal connections between the LEC and MEC (for review, see van Strien et al., 2009). Experimental work suggests that homologous pathways may be present in humans: for example, Maass et al. (2015) defined the alERC and pmERC based on their functional connectivity to the PRC and PHC, respectively. Because this protocol derived the alERC's boundaries through functional connectivity with the PRC (paralleling LEC-PRC connections in rodents and monkeys), we argue that it is a more precise human analog to the rodent LEC than previously used definitions of the human LEC, which involved simple geometric division of the ERC.

On the same anatomical basis, the PMAT (posterior medial, anterior temporal) framework proposes that the alERC and the PRC form the hub of a network relating representations of specific entities (e.g., objects/faces) to their associated saliences and existing semantic concepts (Ranganath and Ritchey, 2012; Ritchey et al., 2015). A theoretically aligned view, the representational-hierarchical model, holds that the PRC and, by extension, the adjoining alERC, sits at the apex of the ventral visual stream, an object-processing pathway of increasingly complex representations. Relatively simple object features are represented in posterior regions and more complex conjunctions of object features (at approximately the level of an object) are represented in the PRC and likely the neighboring alERC (Cowell et al., 2010; Saksida and Bussey, 2010; Barense et al., 2012; Erez et al., 2016).

Consistent with these models, the two previous cognitive studies investigating the human LEC (using fMRI in healthy young adults) showed increased LEC activation when distinguishing faces/objects from lures with similar features (Schultz et al., 2012; Reagh and Yassa, 2014). In contrast, and possibly reflecting reciprocal connections with the spatially responsive MEC/hippocampus, rodent studies suggest that the LEC may be involved in representing the spatial properties of objects $(\mathrm{Kn}-$ ierim et al., 2014). Direct recording studies report LEC place cells that fired at the location of novel objects (Deshmukh and Knierim, 2011) or at locations where objects were located previously (Tsao et al., 2013).

Connecting the theoretical models with the rodent literature, we reasoned that the alERC may be involved in processing the conjunctive arrangement of the parts of an object (i.e., intra-item configural processing, which may be considered one of many spatial properties of an object). To assess the effect of alERC volume differences on configural processing, we designed a novel eye-tracking paradigm, tested a group of older adult participants at varying stages of decline, and used a recently developed method to quantify the volume of their alERC (Maass et al., 2015) and surrounding MTL cortical regions and hippocampal subfields (Olsen et al., 2013).

\section{Materials and Methods Participants}

Thirty-eight community-dwelling older adults were recruited from the community in Toronto. Data from three participants were excluded due to eye-tracker failure. The remaining participants had an average age of 71.7 years (SD: 5.2, range: $58-81,27$ women). Participants had previously received the Montreal Cognitive Assessment (MoCA) (Nasreddine et al., 2005) within the last 22 months (M: 10.4, SD: 6, range: 0-22) and were selected to provide a distribution of MoCA scores (M: 25.7, SD: 2.5, range: $21-30$ ). The MoCA is sensitive to the presence of mild cognitive impairment, which is associated with MTL/hippocampus volume loss (Jack et al., 1997). Our intention was to select for a participant group who had a good distribution of cognitive abilities and MTL/hippocampal regional volumes. These participants were a subset of an original sample of 40 participants who were chosen such that 20 had scored above the MoCA cutoff score $(\geq 26)$ and 20 had scored below it $(<25)$ (Olsen, Yeung et al., 2017). Of the 35 participants whose data we report here, 16 scored above the MoCA cutoff score and 19 scored below it. These two groups were matched for age (original sample: $t_{(38)}=1.29, p=0.20 ; 35$ participants in this study: $t_{(33)}=0.51, p=0.61$ ) and years of education (original sample: $t_{(38)}=0.51, p=0.61 ; 35$ participants in this study: $t_{(33)}=$ $0.20, p=0.84$ ). Despite our attempt to match participants above and below the MoCA cutoff score in terms of demographic characteristics, MoCA and age remained marginally correlated among the 35 participants in this study $(r=-0.289, p=0.07)$. For the purposes of the present study, we were primarily interested in how MTL volume differences related to cognitive performance rather than how participants who scored above/below the MoCA threshold differed; therefore, for all subsequent analyses, we treated all the participants as a single group.

Participants received a battery of neuropsychological tests to further characterize their cognitive status. The battery consisted of the Logical Memory subtest from the Wechsler Memory Scale, 4th Ed. (Wechsler, 2009), Trails A and B (Reitan and Wolfson, 1985), the Digit Span subtest from the Wechsler Adult Intelligence Scale, 4th Ed. (Wechsler, 2008), the Rey-Osterrieth Complex Figure Test (Osterreith, 1944), the Weschler Abbreviated Scale of Intelligence (Wechsler, 1999), and the Visual Object and Space Perception battery (Warrington and James, 1991). Results of the neuropsychological battery are given in Table 1. All participants had normal or corrected-to-normal vision (with glasses or bifocals) and were screened for color blindness, psychological or neurological disorders, brain damage (i.e., stroke or surgery), and metal implants or health conditions that would have precluded MRI. All participants gave informed consent. This research received ethical approval from the Research Ethics Board of the University of Toronto.

\section{MRI scan parameters}

High-resolution T2-weighted images were acquired in an oblique-coronal plane perpendicular to the long axis of the hippocampus (TE/TR = $68 \mathrm{~ms} / 3000 \mathrm{~ms}, 20-28$ slices depending on head size, $512 \times 512$ acquisition matrix, voxel size $=0.43 \times 0.43 \times 3 \mathrm{~mm}$, no skip, FOV $=220$ $\mathrm{mm}$ ), on a 3 T Siemens Trio scanner at the Rotman Research Institute at Baycrest (Toronto, Ontario). The first slice was placed anterior to the appearance of the collateral sulcus (including the temporal pole where possible) and the last posterior to the hippocampal tail to ensure full coverage of the entire hippocampus and all of the MTL cortices included in the volumetric analyses for all participants. To confirm slice placement, a T1-weighted MP-RAGE whole-brain anatomical scan (TE/TR = $2.63 \mathrm{~ms} / 2000 \mathrm{~ms}, 176$ slices perpendicular to the AC-PC line, $256 \times 192$ acquisition matrix, voxel size $=1 \times 1 \times 1 \mathrm{~mm}$, FOV $=256 \mathrm{~mm}$ ) was acquired immediately before the T2-weighted scan. The T1-weighted images were also used to estimate total intracranial volume for head-size correction (see "Volume correction for head size" section below).

\section{Manual segmentation}

For each participant, L.-K.Y. manually segmented three hippocampal subfields (CA1, dentate gyrus/CA2 and 3, and subiculum) and four MTL cortices (alERC, pmERC, PRC, and PHC) on coronal slices of the T2wighted structural scans (in-plane resolution: $0.43 \times 0.43 \mathrm{~mm}, 3 \mathrm{~mm}$ between slices) using FSLview (version 3.1). Manual segmentation followed the Olsen-Amaral-Palombo (OAP) protocol (Olsen et al., 2013; Palombo et al., 2013; see also the appendix to Yushkevich et al., 2015a) supplemented with a modified version of the protocol provided by Maass 
Table 1. Neuropsychological battery results, means (SD)

\begin{tabular}{|c|c|c|}
\hline Test & $\begin{array}{l}\text { All participants } \\
(N=38)\end{array}$ & $\begin{array}{l}\text { Participants included } \\
\text { in data analysis } \\
(n=35)\end{array}$ \\
\hline \multirow[t]{2}{*}{$\operatorname{MoCA}(/ 30)$} & $25.4(2.8)$ & $25.7(2.5)$ \\
\hline & Slightly impaired & Slightly impaired \\
\hline \multicolumn{3}{|l|}{ WMS-IV logical memory } \\
\hline \multirow{2}{*}{ Immediate recall scaled score (/20) } & $11.3(2.7)$ & $11.2(2.7)$ \\
\hline & 64.2 nd percentile & 63.0rd percentile \\
\hline \multirow{2}{*}{ Delayed recall scaled score $(/ 20)$} & $10.8(2.6)$ & $10.7(2.6)$ \\
\hline & 58.2nd percentile & 57.4th percentile \\
\hline Recognition accuracy & $82.1 \%(10.4 \%)$ & $81.6 \%(10.3 \%)$ \\
\hline \multirow[t]{2}{*}{ Trails A } & $42.9 \mathrm{~s}(13.8 \mathrm{~s})$ & $42.9 \mathrm{~s}(13.5 \mathrm{~s})$ \\
\hline & 42.2nd percentile & 42.0nd percentile \\
\hline \multirow[t]{2}{*}{ Trails B } & $91.9 \mathrm{~s}(35.6 \mathrm{~s})$ & $90.4 \mathrm{~s}(34.7 \mathrm{~s})$ \\
\hline & 56.6th percentile & 56.9th percentile \\
\hline \multirow[t]{2}{*}{ Digit span forward score (/16) } & $10.2(2.2)$ & $10.2(2.2)$ \\
\hline & 52.0nd percentile & 52.3rd percentile \\
\hline \multirow[t]{2}{*}{ Digit span backward score (/14) } & $6.8(2.5)$ & $6.7(2.4)$ \\
\hline & 33.5th percentile & 31.9th percentile \\
\hline \multicolumn{3}{|l|}{ Rey-0sterrieth complex figure } \\
\hline \multirow[t]{2}{*}{ Copy (/32) } & $26.8(5.4)$ & $26.9(5.6)$ \\
\hline & 26.7th percentile & 28.2nd percentile \\
\hline \multirow{2}{*}{ Immediate recall (/32) } & $12.1(6.6)$ & $12.2(6.6)$ \\
\hline & 40.0th percentile & 40.6th percentile \\
\hline \multirow[t]{2}{*}{ Delayed recall (/32) } & $10.6(6.4)$ & $10.9(6.5)$ \\
\hline & 33.5th percentile & 35.5th percentile \\
\hline \multicolumn{3}{|l|}{ Weschler Abbreviated Scale of Intelligence } \\
\hline \multirow[t]{2}{*}{ Vocabulary (/80) } & $59.4(9.8)$ & $59.5(10.2)$ \\
\hline & 66.2 nd percentile & 66.5th percentile \\
\hline \multirow[t]{2}{*}{ Similarities (/48) } & $36.3(5.1)$ & $36.5(5.2)$ \\
\hline & 75.3rd percentile & 75.8th percentile \\
\hline \multirow{2}{*}{ Matrix reasoning (/32) } & $21.9(6.8)$ & $22.1(6.7)$ \\
\hline & 75.7th percentile & 76.6th percentile \\
\hline \multirow[t]{2}{*}{ Block design (/71) } & $29.9(14.9)$ & $30.5(15.4)$ \\
\hline & 54.2nd percentile & 54.9th percentile \\
\hline \multicolumn{3}{|l|}{ Visual Object and Spatial Perception Battery } \\
\hline Shape detection (/20) & $19.2(1.1)$ & $19.2(1.2)$ \\
\hline (Cut-off score $<15$ ) & Pass & Pass \\
\hline Incomplete letters (/20) & $19.3(0.8)$ & $19.3(0.8)$ \\
\hline (Cut-off score $<16$ ) & Pass & Pass \\
\hline Dot counting $(/ 10)$ & $9.8(0.4)$ & $9.9(0.4)$ \\
\hline (Cut-off score $<8$ ) & Pass & Pass \\
\hline Position discrimination (/20) & $19.3(1.6)$ & $19.2(1.6)$ \\
\hline (Cut-off score $<18$ ) & Pass & Pass \\
\hline Number location (/10) & $9.1(1.6)$ & $9.2(1.6)$ \\
\hline (Cut-off score <7) & Pass & Pass \\
\hline Cube analysis (/10) & $9.4(1.3)$ & $9.3(1.3)$ \\
\hline (Cut-off score $<6$ ) & Pass & Pass \\
\hline Silhouettes $(/ 30)$ & $19.8(5.2)$ & $19.7(5.4)$ \\
\hline (Cut-off score $<15$ ) & Pass & Pass \\
\hline Object decision (/20) & $16.9(2.0)$ & $16.8(2.0)$ \\
\hline (Cut-off score <14) & Pass & Pass \\
\hline Progressive silhouettes (/20) & $10.2(3.1)$ & $10.2(3.2)$ \\
\hline (Cut-off score $>15$ ) & Pass & Pass \\
\hline Subjective memory rating ${ }^{\text {a }}$ & $295.1(53.1)$ & $294.2(55.5)$ \\
\hline (Memory functioning questionnaire, /448) & $\begin{array}{l}\text { Minimal subjective } \\
\text { difficulties }\end{array}$ & $\begin{array}{l}\text { Minimal subjective } \\
\text { difficulties }\end{array}$ \\
\hline
\end{tabular}

Maximum and cut-off scores for tests are indicated in parentheses in left column. WMS-IV, Wechsler Memory Scale, Ed 4.

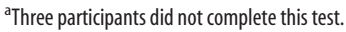

et al. (2015) for the subdivisions of the ERC (see Figure 1 for a visualization of the segmentation protocol).

We considered these particular regions for two reasons. First, because these regions are directly connected to the alERC (Suzuki and Amaral, 1994; Burwell, 2000), we wished to explore whether any observed alERCbehavior correlations were mediated by its inputs and outputs. Second, these regions support related cognitive functions/representations that may aid performance. The hippocampus (particularly the trisynaptic loop formed by DG-CA3-CA1) is implicated in pattern separation/completion processes necessary to disambiguate similar stimuli (Norman and O'Reilly, 2003; Rolls, 2016). The PRC, PHC, and hippocampus are theorized to support object/context representations used in memory and perception (Cowell et al., 2010; Barense et al., 2012; Ranganath and Ritchey, 2012).

The OAP protocol follows the guidelines of Insausti et al. (1998) for delineating the ERC and PRC and the guidelines of Pruessner et al. (2002) for delineating the PHC. The ERC was further subdivided into the alERC and the pmERC following the protocol of Maass et al. (2015), which was based on functional connectivity with the PRC and PHC, respectively. See Olsen, Yeung et al. (2017) for a more extensive description of the alERC/pmERC boundary used in our segmentation protocol. We note that, because we followed the protocol of Insausti et al. (1998) to define the lateral edge of the ERC, the resulting lateral boundary of the alERC/ pmERC regions in our protocol differs slightly from that of Maass et al. (2015). Our alERC/pmERC regions extend into the collateral sulcus when the depth of the collateral sulcus is "shallow" (depth $<1 \mathrm{~cm}$ ) or "regular" (depth between 1 and $1.5 \mathrm{~cm}$ ). As a result, the alERC/pmERC subregions defined here overlap with the trans entorhinal region defined by Braak and Braak $(1991,1992)$ and also with the medial PRC regions used elsewhere in the literature (Krumm et al., 2016; Wolk et al., 2017).

Following the OAP protocol, hippocampal subfield segmentations were based on Amaral and Insausti (1990). The strata radiatum, lacunosum, and moleculare (SLRM) was used to divide the subiculum and CA1 from the CA2/3/dentate gyrus. The internal boundaries between CA1 and the other two regions are detailed in Figure 1 of Palombo et al. (2013). The OAP protocol (developed for use in structural scans of younger adults) typically includes two additional ROIs, which cover the anterior head and the posterior tail of the hippocampus, where the organization of the subfields is more complex and the SLRM is sometimes too indistinct to differentiate hippocampal subfields. There is currently little consensus as to how to subdivide these regions into subfields using in vivo $3 \mathrm{~T}$ MRI, which is why it has been our practice to combine them into a single ROI, as do other high-resolution protocols (e.g., see Schlicting and Preston protocol in Yushkevich et al., 2015a). Because the hippocampal subfields within these regions were not segmented into subregions, these regions were excluded from further analysis.

Average volumes for each manually segmented brain region are presented in Table 2 and correlations between brain region volumes are presented in Table 3.

\section{Intra-rater and inter-rater segmentation reliability}

Intra-rater reliability was established by comparing the segmentation of five randomly selected scans, completed by the same rater (L.-K.Y.) after a delay of 1-4 months. Inter-rater reliability was evaluated by comparing the segmentation of five randomly selected scans by a second rater (R.K.O) to those of L.-K.Y. Both authors were blinded to MoCA score, task performance, and the identities of participants until after all manual segmentation (including inter-rater and intra-rater reliability) was completed. Reliability was assessed using the intra-class correlation coefficient (ICC, which evaluates volume reliability; Shrout and Fleiss, 1979) and the Dice metric (which also takes spatial overlap into account; Dice, 1945), computed separately for each region in each hemisphere. ICC $(3, \mathrm{k})$ was computed for intra-rater reliability (consistency) and $\operatorname{ICC}(2, \mathrm{k})$ was computed for inter-rater reliability (agreement). Dice was derived using the formula $2 *$ (area of intersecting region)/(area of original segmentation + area of repeat segmentation); a Dice overlap metric of 0 represents no overlap, whereas a metric of 1 represents perfect overlap. Intra-rater and inter-rater reliability results are shown in Table 4 . These scores are comparable to reliability values reported previously for manual segmentation of hippocampal subfields and MTL cortices (Wisse et al., 2012; Yushkevich et al., 2015b) and are consistent with our previous work (Olsen et al., 2013; Palombo et al., 2013). 


\section{Volume correction for head size}

All manually segmented region volumes were corrected for head size using a regressionbased method to account for differences in brain size between participants. Estimated total intracranial volume (eTIV) was derived using FreeSurfer (version 5.3) (Buckner et al., 2004). By regressing the volume of each region with eTIV, a regression slope $\beta$ was obtained for each region (representing the effect of eTIV change on that region's volume). Then, the volume of each region was adjusted by that participant's eTIV using the following formula:

$\mathrm{Vol}_{\mathrm{adj}}=\mathrm{Vol}_{\mathrm{raw}}+\beta\left(\mathrm{eTIV}_{\text {participant }}-\mathrm{eTIV}_{\text {mean }}\right)$

The head size correction was computed separately for each region in each hemisphere. Volumes were subsequently summed in each region across the two hemispheres, giving a single volume for each region for each participant.

\section{Eye-tracking task}

We developed a novel eye-tracking paradigm assessing intra-item configural processing of conjunctive objects at varying degrees of novelty and investigated whether performance was affected by volumetric differences in the alERC and other MTL regions (Fig. 2A). Participants incidentally viewed individual computer-generated conjunctive objects (comprised of distinct upper and lower halves presented on a gray background; Fig. $2 B$ ) for $5 \mathrm{~s}$ while their viewing of three equally sized ROIs on the objects (top, middle, and bottom) was recorded using EyeLink 1000/II eye trackers (SR Research). Each participant completed 16 blocks, each containing 21 trials (each block used entirely unique stimuli). Within each block, three objects were repeatedly presented six times each. On the seventh repetition, we assessed the effect of novelty for whole objects (versus parts of objects) by presenting one object from each of three novelty conditions: (1) a repeated object identical to an item previously presented in the same block; (2) a recombined object in which each of the two halves had been presented previously as parts of different objects in the block; and (3) a novel object in which both halves were new. After each trial, participants were asked to rate how well the two parts of the stimulus fit together (on a scale of 1-3) to encourage holistic viewing of the conjunctive objects. No time limit was imposed on answering this question and eye movements were not recorded during this time. Across participants, we counterbalanced for which set of objects were used in each novelty condition.

We defined two primary and three ancillary eye-tracking-based outcome variables for each novelty condition (Fig. 2C). Our first primary outcome variable was the proportion of fixations to the middle ROI (which contained the "join" between the upper and lower halves of the object). Because the middle ROI contained information that would allow participants to distinguish successfully between repeated objects (familiar features in a familiar configuration) and recombined objects (familiar features in a novel configuration), differences in viewing to the middle ROI were taken to reflect differences in configural processing for a particular object. As shown in Figure 2B, this was calculated by dividing the number of fixations made to the middle ROI by the total number of fixations made to the whole object on a per-trial basis and then averaging over all the trials in each novelty condition (repeated, recombined, novel). To avoid skewing the results with trials in which there were too few fixations (where the denominator is very small, leading to larger variability in the proportion measure), we excluded all trials with $<5$ fixations on the object from this analysis; this accounted for only $\sim 1.8 \%$ of trials overall.

Our second primary outcome variable was the normalized number of fixations to the entire object. This was calculated by taking the average number of fixations made to the objects for all the trials in a given novelty
Table 2. Average volumes for manually segmented brain regions

\begin{tabular}{lcc}
\hline Brain region & $\begin{array}{l}\text { All participants } \\
(N=38)\end{array}$ & $\begin{array}{l}\text { Participants included in } \\
\text { data analysis }(n=35)\end{array}$ \\
\hline $\begin{array}{l}\text { Hippocampus } \\
\text { CA1 }\end{array}$ & $1244.71 \pm 157.41$ & $1242.33 \pm 159.86$ \\
Subiculum & $1140.85 \pm 217.08$ & $1120.39 \pm 198.20$ \\
DG/CA23 & $1952.49 \pm 344.71$ & $1959.34 \pm 354.68$ \\
MTL & & \\
PRC & $4804.26 \pm 1193.44$ & $4833.72 \pm 1232.25$ \\
alERC & $1367.08 \pm 267.85$ & $1374.44 \pm 273.98$ \\
PmERC & $454.36 \pm 111.99$ & $452.52 \pm 116.05$ \\
PHC & $3664.36 \pm 633.67$ & $3661.96 \pm 653.84$ \\
\hline
\end{tabular}

Average volumes $\left(\mathrm{mm}^{3} \pm \mathrm{SD}\right)$ for each of the three manually segmented hippocampal subfields and four MTL cortices segmented for this study (corrected for head size).

Table 3. Correlations between volumes of manually segmented brain regions

\begin{tabular}{llllllll}
\hline & CA1 & Subiculum & DG/CA23 & PRC & alERC & pmERC & PHC \\
\hline CA1 & 1 & $0.375^{*}$ & $0.671^{* *}$ & $0.366^{*}$ & $0.318^{*}$ & $0.347^{*}$ & $0.440^{* *}$ \\
Subiculum & 1 & 0.162 & -0.272 & -0.015 & $0.590^{* *}$ & 0.228 \\
DG/CA23 & & & 1 & $0.468^{* *}$ & 0.258 & 0.219 & 0.311 \\
PRC & & & & 1 & $0.395^{*}$ & 0.011 & 0.159 \\
alERC & & & & & 1 & 0.198 & 0.201 \\
PmERC & & & & & 1 & 0.274 \\
PHC & & & & & & & 1
\end{tabular}

Pearson's correlations between the volumes of all manually segmented regions; ${ }^{*} p<0.05,{ }^{* *} p<0.01$.

condition (repeated, recombined, or novel) and normalizing by the average number of fixations made to all the objects during their initial presentation in the first repetition. The number of fixations made to objects during the first repetition within each block (when all the objects were entirely novel) served as a baseline for how many fixations that a particular participant would make to an entirely novel object. This followed the procedure that we used in our previous work (Yeung et al., 2013) to derive a normalized eye-tracking-based measure of novelty that controlled for absolute differences in the number of fixations between 
Table 4. Inter-rater and intra-rater reliability measurements for manual segmentation

\begin{tabular}{|c|c|c|c|c|c|c|c|c|}
\hline \multirow[b]{2}{*}{ Subregion } & \multicolumn{2}{|c|}{$\begin{array}{l}\text { Intra-rater: } \\
\text { Dice }\end{array}$} & \multicolumn{2}{|c|}{$\begin{array}{l}\text { Intra-rater: } \\
\text { ICC }\end{array}$} & \multicolumn{2}{|c|}{$\begin{array}{l}\text { Inter-rater: } \\
\text { Dice }\end{array}$} & \multicolumn{2}{|c|}{$\begin{array}{l}\text { Inter-rater: } \\
\text { ICC }\end{array}$} \\
\hline & Left & Right & Left & Right & Left & Right & Left & Right \\
\hline CA1 & 0.88 & 0.87 & 0.94 & 0.95 & 0.74 & 0.66 & 0.92 & 0.91 \\
\hline Subiculum & 0.85 & 0.84 & 0.89 & 0.88 & 0.67 & 0.66 & 0.81 & 0.85 \\
\hline $\mathrm{DG} / \mathrm{CA} 23$ & 0.91 & 0.90 & 0.94 & 0.99 & 0.75 & 0.73 & 0.91 & 0.96 \\
\hline alERC & 0.86 & 0.85 & 0.96 & 0.86 & 0.72 & 0.73 & 0.87 & 0.71 \\
\hline pmERC & 0.82 & 0.80 & 0.90 & 0.86 & 0.59 & 0.64 & 0.95 & 0.80 \\
\hline PRC & 0.87 & 0.89 & 0.98 & 0.91 & 0.74 & 0.76 & 0.98 & 0.99 \\
\hline PHC & 0.86 & 0.84 & 0.89 & 0.95 & 0.71 & 0.77 & 0.86 & 0.96 \\
\hline
\end{tabular}

Dice was computed for both intra-rater and inter-rater agreement. ICC $(3, \mathrm{k})$ was calculated for intra-rater and ICC $(2, \mathrm{k})$ was computed for inter-rater reliability.

participants. Because more fixations are made to novel objects compared with previously viewed objects (Althoff and Cohen, 1999), this variable serves as a measure of novelty detection for the object as a whole. A score of 1 or more here indicates that objects in a given novelty condition were being treated as novel (i.e., the same number of fixations were made compared with when the object was entirely novel during the first presentation), whereas a score of $<1$ indicates that the objects were being treated as though they had been seen previously (i.e., fewer fixations were made compared with when the object was entirely novel).

To further explore changes in the proportion of fixations directed to the middle ROI across novelty conditions, we derived three ancillary eye-tracking measures: the normalized number of fixations to the middle ROI, the normalized number of fixations to the peripheral ROIs, and the number of transitions between ROIs. The first two ancillary measures used the same normalization method used for the normalized number of fixations to the entire object. For both the middle and peripheral ROIs, the average number of fixations made to those respective ROIs in all of the trials of a certain novelty condition was normalized by the average number of fixations made to the entire objects shown in the first repetition (again, serving as a baseline for the number of fixations made to an entirely novel object). As a result of how they were calculated, the normalized number of fixations to the middle and peripheral ROIs necessarily sum to the normalized number of fixations to the entire object. Note that the normalized number of fixations to the middle ROI is distinct and different from the proportion of fixations to the middle ROI. The former is a count of how many fixations were made to the middle ROI, serving as a measure of novelty detection. In contrast, the latter describes the relative distribution of fixations across the object and serves as a measure of configural processing (i.e., the first primary outcome measure described above). The number of transitions between ROIs counted the number of saccades that moved between different parts of the conjunctive objects. In the context of this study, these saccades may reflect spatial processing necessary to bind together different object halves into a coherent object representation.

\section{Stimulus and ROI properties}

The conjunctive objects comprised upper and lower halves. In the recombined condition, object halves retained their relative locations from when they were first presented (i.e., an object half that had appeared previously as an upper half stayed as an upper half in the recombined object). The objects varied in width from 264 to 564 pixels (M: 346.6 pixels, SD: 50.0 pixels) subtending $25.8-55.1 \%$ of the screen horizontally (M: 33.9\%, SD: $4.9 \%$ ) and covering a horizontal visual angle of 9.6-20.4 (M: $12.6^{\circ}, \mathrm{SD}: 1.8^{\circ}$ ). In height, the objects varied from 287 to 602 pixels (M: 470.1 pixels, SD: 61.3 pixels), subtending $37.3-78.3 \%$ of the screen vertically (M: $61.2 \%$, SD: $8.0 \%)$ and covering a vertical visual angle of $11.6-24.1^{\circ}\left(\mathrm{M}: 18.6^{\circ}, \mathrm{SD}: 2.5^{\circ}\right)$.

The location of each stimulus was jittered pseudorandomly so that the center of the stimulus was not always presented at the center of the screen (Fig. 2B). In all cases, the entire stimulus appeared on the screen (i.e., the jittering did not cause any stimuli to be cut off by the edge of the screen). Despite jittering our stimuli in this fashion, the first fixation still fell on the middle ROI on $68.6 \%$ of trials while falling on the peripheral ROIs on
$15.2 \%$ of trials and off the object entirely for $16.1 \%$ of trials (this results from the relatively large size of the stimuli and the requirement that the entire stimuli stay on the screen, constraining the number of possible locations it could take). To address the concern that this might have caused a bias in our results, we have removed the first fixation on every trial from all our analyses. The ROIs were defined based on the size of each individual stimulus. All three ROIs spanned the entire width of each stimulus and each ROI covered exactly one-third of the vertical extent of each stimulus (as depicted in Fig. $2 B$ ). Notably, these ROIs excluded the rest of the screen outside of each stimulus.

\section{Eye-tracker setup}

The experimental task was presented on a 21.2-inch monitor $(36 \times 30$ $\mathrm{cm})$ at a resolution of $1024 \times 768$ pixels using Experiment Builder (SR Research). For 25 participants, eye-tracking measures were recorded using an EyeLink II head-mounted eye tracker; for the remaining 13 participants, eye-tracking measures were recorded using an EyeLink 1000 desktop-mounted eye tracker. Repeated-measures ANOVAs showed that there was no main effect of the eye-tracker model in terms of fixations per trial $\left(F_{(1,34)}=0.88, p=0.35\right)$ or in terms of the proportion of fixations made to the middle ROI $\left(F_{(1,34)}=0.16, p=0.69\right)$. There was also no interaction of eye-tracker model and condition either in terms of fixations per trial $\left(F_{(2,68)}=0.80, p=0.45\right)$ or in the proportion of fixations made to the middle $\operatorname{ROI}\left(F_{(2,68)}=0.04, p=0.96\right)$. Accordingly, all analyses were collapsed across the eye-tracker model used.

The EyeLink 1000 sampled at a rate of $1000 \mathrm{~Hz}$ with a spatial resolution of $0.01^{\circ}$ and an accuracy of $0.25^{\circ}$, whereas the EyeLink II sampled at a rate of $500 \mathrm{~Hz}$ with a spatial resolution of $0.01^{\circ}$ and an accuracy of $0.5^{\circ}$. Participants were positioned $55 \mathrm{~cm}$ away from the monitor; participants using the EyeLink 1000 placed their heads on a chin rest to limit head motion, which was unnecessary with the EyeLink II system because it corrects for head motion. Nine-point calibration was performed before testing, and was repeated until the average gaze error was $<1^{\circ}$, with no point having a gaze error exceeding $1.5^{\circ}$. Before each trial, drift correction was performed (which causes the initial fixation on each trial to be focused at the center of the screen), with 9-point calibration being repeated if drift error exceeded $2^{\circ}$.

\section{Statistical analysis}

Repeated-measures ANOVAs and planned paired-samples $t$ tests were used to identify differences in each of the primary outcome variables (proportion of fixations to the middle ROI and normalized number of fixations to the entire object) in each novelty condition (i.e., only considering novel, recombined, and repeated trials shown during the seventh repetition). To further clarify the differences in the primary outcome variables across different novelty conditions, we performed pairedsamples $t$ tests on the ancillary outcome variables (normalized number of fixations to middle ROI, normalized number of fixations to peripheral ROIs, and number of transitions between ROIs). Theses ancillary variables merely reflected aspects of the primary outcome variables and were not truly independent from them (e.g., the normalized number of fixations to the entire object is the sum of the normalized number of fixations to the middle and peripheral ROIs); therefore, they were not included in the subsequent volumetric analyses.

To assess the importance of each brain region on the primary outcome variables only, multiple regression was used with each outcome variable as the dependent variable and the volumes of the seven brain regions as predictors. For brain regions that significantly predicted task performance, Steiger's $Z$ test was used to explore differences in their correlations across novelty conditions. Additional multiple regression analyses evaluated whether the proportion of fixations directed to the middle $\mathrm{ROI}$ in each novelty condition predicted variance in alERC volume unaccounted for by MoCA or age. All statistical tests were two-tailed and conducted at $\alpha=0.05$. Mauchly's test of sphericity was applied to repeated-measures ANOVAs; when the assumption of sphericity was violated, the Greenhouse-Geisser correction was applied. Multiple regressions were tested for multicollinearity; residual plots were inspected to check for nonlinearity and heteroscedasticity. 


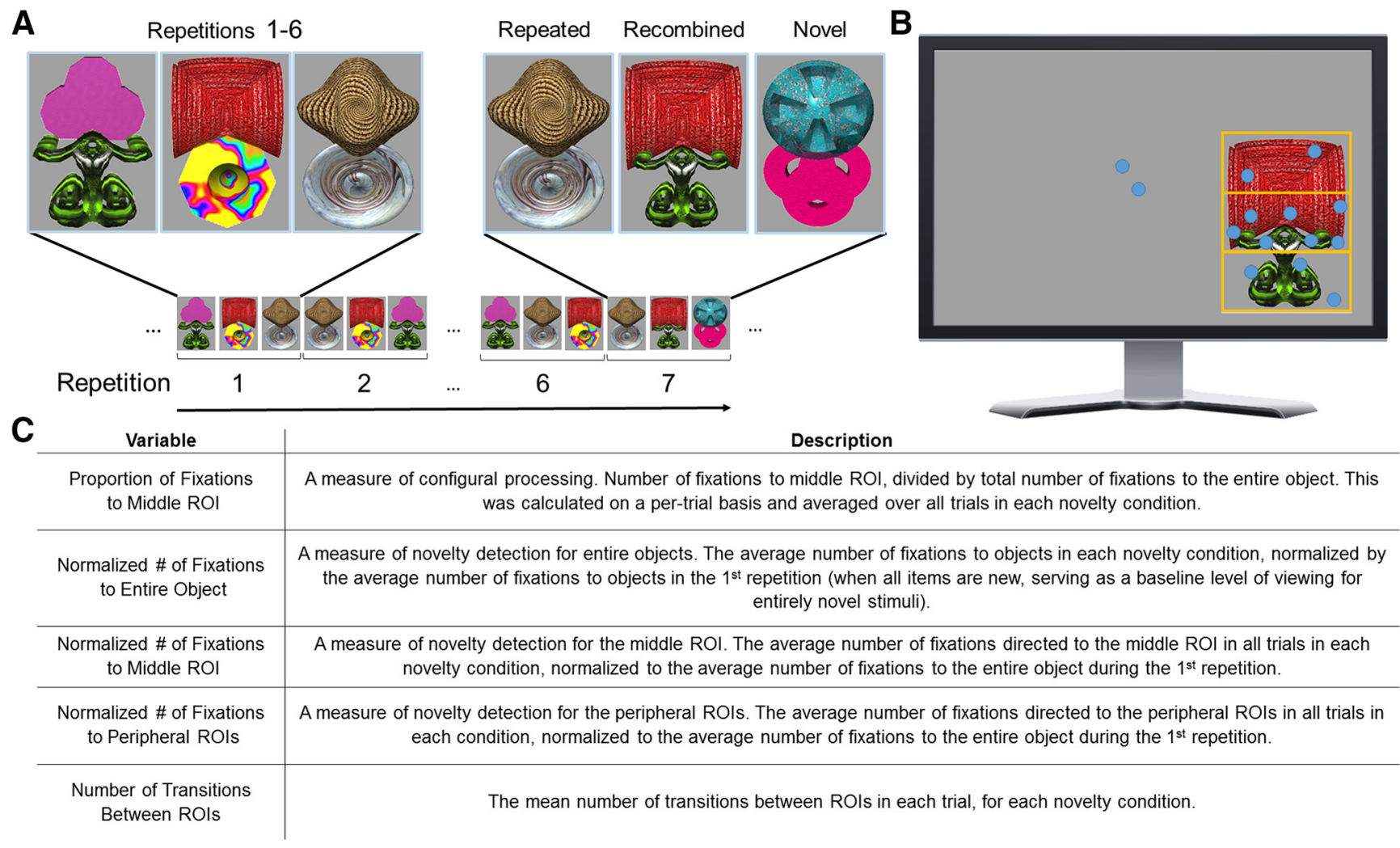

Figure 2. Behavioral task used in this study. A, Diagram showing the task design. Participants performed passive viewing of individual configural 2-part object stimuli for $5 \mathrm{~s}$ each. 0 nly one block (of 16 in the study) is depicted in the figure. Each block contained six repetitions of the same set of three objects, followed by a seventh repetition with three critical trials, that included the following: (1) a repeated object identical to one shown during the first six repetitions; (2) an object comprising recombined parts of previously viewed objects; and (3) an entirely novel object. The ordering of these objects was randomized across blocks and no objects or parts of objects were repeated across blocks. $B$, Example of a single trial. Note that the presented object is not centered on the display; rather, its location changed from trial to trial. Viewing to three equally sized ROls (top, middle, and bottom; shown here in yellow) distributed vertically were recorded; fixations are shown as blue circles here (note that the ROIs and fixations are presented here for display purposes only and were not visible to participants during the study). Viewing to the middle ROI (which contained the intersection of the two parts) was of special interest for evaluating intra-item configural processing because it contains the critical "join" between the two halves of the conjunctive objects, which allows recombined objects to be distinguished from repeated objects. In this example, there are seven fixations to the middle ROI, 12 fixations to the whole object, and 14 fixations overall. Therefore, the proportion of fixations directed to the middle ROI for this particular trial is $7 / 12=0.583$. C, List of the primary and ancillary eye-tracking-based outcome variables used in this study.

\section{Results}

\section{Behavioral results}

We ran repeated-measures ANOVAs investigating the effect of novelty condition (repeated, recombined, and novel) on the proportion of fixations directed to the middle ROI and the normalized number of fixations to the entire object (our two primary eye-tracking measures). These showed main effects of novelty condition for the proportion of fixations directed to the middle $\operatorname{ROI}\left(F_{(2,68)}=11.83, p=1.58 \times 10^{-4}, \eta^{2}=0.26\right)$, as well as the normalized number of fixations to the entire object $\left(F_{(2,68)}=\right.$ $62.15, p=4.45 \times 10^{-16}, \eta^{2}=0.65$ ) (Fig. 3). Mauchly's test indicated the assumption of sphericity was violated for the ANOVA on the proportion of fixations to the middle $\mathrm{ROI}\left(\chi^{2}(2)=8.88\right.$, $p=0.01$; Greenhouse-Geisser correction was applied, $\varepsilon=0.81$ ). $t$ tests showed that a greater proportion of fixations were directed to the middle ROI for repeated objects than recombined objects $\left(t_{(34)}=2.99, p=0.005\right)$. In turn, a greater proportion of fixations directed to the middle ROI for recombined objects than novel objects $\left(t_{(34)}=2.20, p=0.03\right.$; Fig. $\left.3 A\right) . t$ tests comparing the normalized number of fixations with the entire object showed fewer fixations to repeated objects than recombined objects $\left(t_{(34)}=\right.$ $\left.7.86, p=3.74 \times 10^{-9}\right)$ and fewer fixations for recombined objects than novel objects $\left(t_{(34)}=3.03, p=0.005\right)$ (Fig. 3B). This pattern matched our previously reported findings (Yeung et al., 2013). Figure $3 C$ shows the unnormalized fixation counts to the entire object, the middle ROI, and the peripheral ROIs.
Using our ancillary outcome measures to investigate the differences in the proportion of fixations to the middle ROI across conditions, we found that this was not driven by differences in the normalized number of fixations directed to the middle ROI (repeated vs recombined: $t_{(34)}=1.88, p=0.07$; recombined vs novel: $t_{(34)}=0.38, p=0.70$; Fig. $4 A$ ). Rather, it is caused by the greater normalized number of fixations directed to the peripheral ROIs in the recombined and novel conditions (repeated vs recombined: $t_{(34)}=6.88, p=6.33 \times 10^{-8}$; recombined vs novel: $t_{(34)}=2.98, p=0.005$; Fig. $4 B$ ).

One explanation for this pattern of fixations is that processing of spatial information in the configurally important middle ROI is necessary for object recognition in all conditions, but recombined objects (which have a novel configuration) and novel objects (which have a novel configuration and novel features) require additional processing of the peripheral features for successful identification. In contrast, viewing to the middle ROI provides sufficient information for successful identification of repeated objects. This interpretation is supported by the data on transitions between ROIs (Fig. 4C): fewer transitions between ROIs were made to the repeated objects compared with objects in the other two novelty conditions (repeated vs recombined: $t_{(34)}=11.26, p=5.17 \times 10^{-13}$, repeated vs novel: $t_{(34)}=13.07$, $\left.p=8.12 \times 10^{-15}\right)$; however, the difference between recombined and novel objects only trended toward significance $\left(t_{(34)}=1.97\right.$, $p=0.057)$. 
A

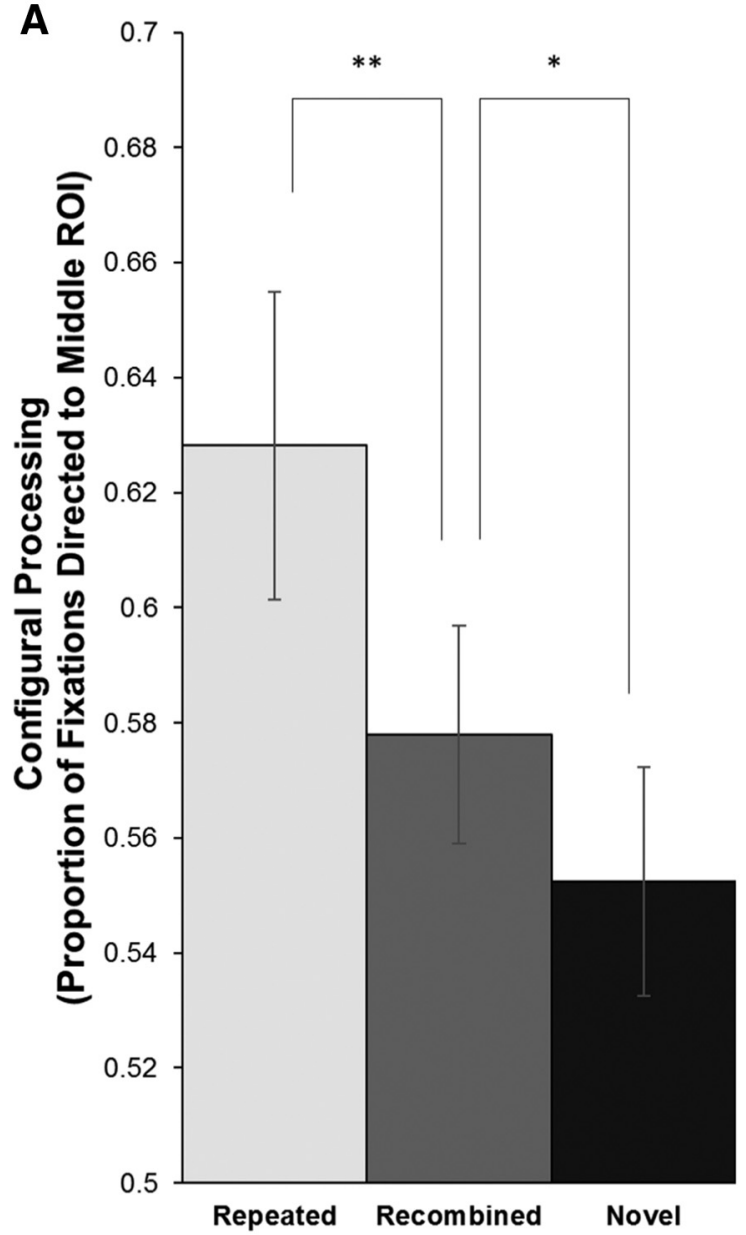

B

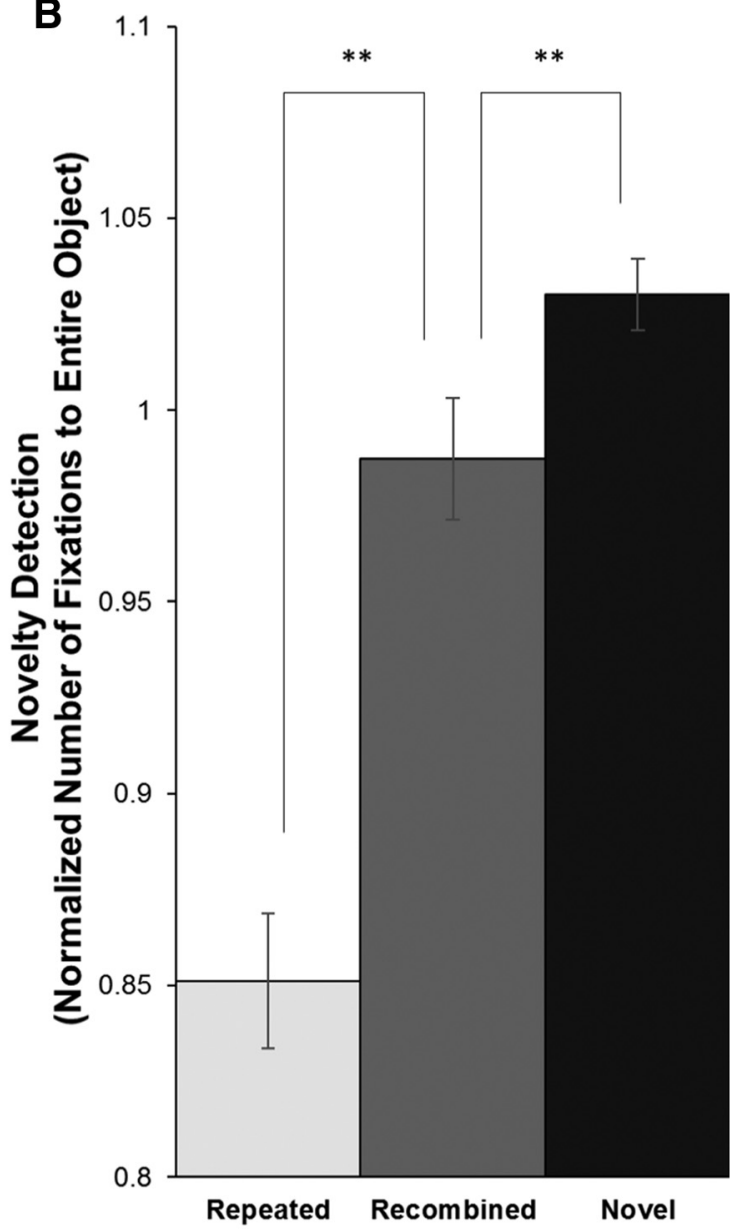

\begin{tabular}{c|c|c|c|c} 
C & Repeated & Recombined & Novel & 1st Repetition \\
\hline Whole Object & $\begin{array}{c}11.5 \\
(7.1-16.7)\end{array}$ & $\begin{array}{c}13.3 \\
(9.8-18.4)\end{array}$ & $\begin{array}{c}13.9 \\
(10.4-19.3)\end{array}$ & $\begin{array}{c}13.6 \\
(9.1-19.0)\end{array}$ \\
\hline Middle ROI & $\begin{array}{c}7.3 \\
(3.1-13.4)\end{array}$ & $\begin{array}{c}7.7 \\
(4.4-11.4)\end{array}$ & $\begin{array}{c}7.7 \\
(3.8-10.8)\end{array}$ & $\begin{array}{c}7.1 \\
(3.6-10.4)\end{array}$ \\
\hline Peripheral ROIs & $\begin{array}{c}4.2 \\
(0.4-9.6)\end{array}$ & $\begin{array}{c}5.7 \\
(2.1-10.3)\end{array}$ & $\begin{array}{c}6.2 \\
(1.6-9.7)\end{array}$ & $\begin{array}{c}6.5 \\
(2.7-10.8)\end{array}$
\end{tabular}

Note that because taking the mean and dividing are not commutative operations, dividing the mean number of fixations presented here in any condition by the mean number of fixations in the 1st repetition will yield a slightly different result than shown in figures $3 \mathrm{~B}, 4 \mathrm{~A}$ and $4 \mathrm{~B}$. The results presented in the bar graphs involve first normalizing (dividing) a participant's number of fixations in a novelty condition (e.g., repeated) by that same participant's number of fixations during the 1 st repetition (thus controlling for individual variability in viewing), then averaging over all participants. The values presented in this table are solely to give a sense of the average fixation count to different ROls under different noveltyconditions.

Figure 3. Behavioral results for primary eye-tracking measures arranged by novelty condition. $A$, Proportion of fixations directed to the middle ROI (a measure of configural processing) relative to the entire object. $\boldsymbol{B}$, Normalized number of fixations to the entire object in each novelty condition. This variable reflects the mean number of fixations to the entire object in a novelty condition normalized by the mean number of fixations made to the entire object during the first repetition (a baseline measure of fixations to entirely novel objects). This serves as a measure of novelty detection controlling for individual variation in the total number of fixations made. ${ }^{* *} p<0.01$. Error bars indicate SEM. C, Mean and range (in parentheses) of raw fixation counts to the whole object and to the middle and peripheral ROls for all three novelty conditions and additionally for the first repetition.

After viewing each conjunctive object for $5 \mathrm{~s}$, participants rated how well the two parts of the object fit together on a scale of 1-3 $(1=$ did not go together well, $3=$ went together well $)$. Paired-samples $t$ tests showed no differences in the fit ratings between repeated (M: 1.62, SD: 0.36) and recombined (M: 1.60, SD: 0.39) objects (repeated vs recombined: $t_{(34)}=0.61, p=0.54$ ). However, novel items (M: 1.75, SD: 0.49 ) had a slightly higher fit rating than items in the other two novelty conditions (novel vs repeated: $t_{(34)}=2.02, p=0.052$; novel vs recombined: $t_{(34)}=$ $2.89, p=0.007)$.

\section{Volumetric imaging results}

Multiple regression analysis was used to assess the influence of MTL/hippocampal subregion volume on the proportion of fixa- 
A

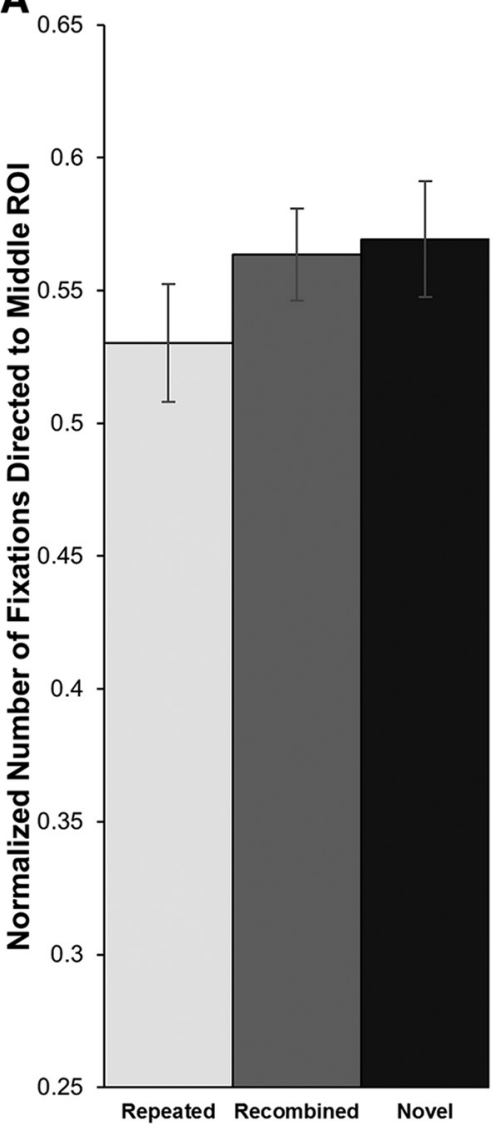

B

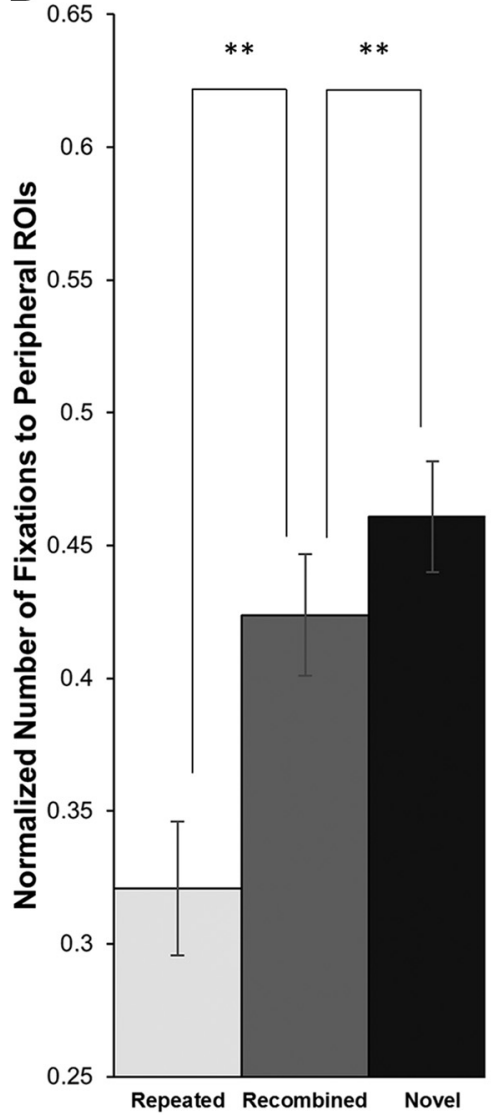

C

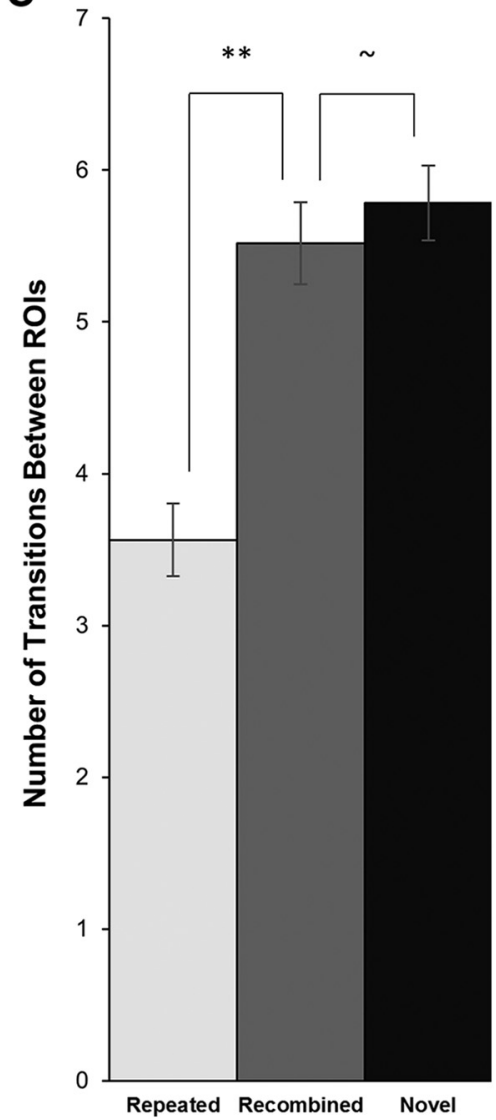

Figure 4. Behavioral results for ancillary eye-tracking measures. $\boldsymbol{A}$, Normalized number of fixations to the middle Rol by novelty condition. $\boldsymbol{B}$, Normalized number of fixations to the peripheral ROls by novelty condition. Both are calculated by taking the mean number of fixations to the respective ROI(s), then normalizing by the mean number of fixations made to the entire object during the first repetition (a baseline measure of fixations to entirely novel objects). This serves as a measure of novelty detection toward each part of the object, controlling for individual variation in the total number of fixations made. $\boldsymbol{C}$, Mean number of transitions between ROIs in each trial by novelty condition. ${ }^{* *} p<0.01$. Error bars indicate SEM.

tions made to the middle ROI (the primary eye-tracking measure of configural processing). Using brain region volumes as predictors revealed that only alERC volume was a significant predictor for the proportion of fixations made to the middle ROI for all three novelty conditions from the seventh repetition (repeated: $t_{(30)}=3.60, p=0.001, \beta=0.60, \mathrm{sr}=0.53$; recombined: $t_{(30)}=$ $4.13, p=3.16 \times 10^{-4}, \beta=0.66$, sr $=0.59$; novel: $t_{(30)}=4.51, p=$ $1.12 \times 10^{-4}, \beta=0.73, \mathrm{sr}=0.65$; Table 5$)$. In other words, greater alERC volume predicted a greater proportion of fixations to the middle ROI regardless of the object's novelty. Figure 5 illustrates this relationship graphically in two different ways: as correlations in Figure $5 A$ and as a median split in Figure $5 B$. This effect holds true even when we consider only the trials in the first six repetitions, treating these trials as though they were an additional novelty condition $\left(t_{(30)}=3.99, p=4.58 \times 10^{-4}, \beta=0.65\right.$, $\mathrm{sr}=$ $0.58)$. Steiger's $Z$ test was used to compare the correlations of the alERC volume residuals (i.e., accounting solely for the contribution of the alERC predictor with the contribution of other regions removed) with proportion of fixations to the middle ROI across the three novelty conditions. This revealed no significant differences (repeated vs recombined: $Z=0.52, p=0.60$; recombined vs novel: $Z=0.79, p=0.43$; repeated vs novel: $Z=1.11, p=$ 0.26). Consistent with recent findings (Olsen et al., 2016), no region was a significant predictor for the normalized number of fixations to the entire object (Table 5), indicating that MTL volume differences did not affect eye movement measures of novelty detection.
To verify that the association between alERC volume and configural processing was not simply driven by global cognitive decline, we investigated whether the proportion of fixations to the middle ROI predicted alERC volume beyond the effect of MoCA or age. Even when MoCA and age were included as predictors in the same model (i.e., having accounted for the variance in alERC volume that they explain), the proportion of fixations to the middle ROI was a significant predictor for alERC volume in all conditions (repeated: $t_{(34)}=$ 3.29, $p=0.002, \beta=0.49$, sr $=0.48$; recombined: $t_{(34)}=4.32, p=$ $1.48 \times 10^{-4}, \beta=0.59, \mathrm{sr}=0.58$; novel: $t_{(34)}=5.25, p=1.04 \times$ $10^{-5}, \beta=0.67$, sr $=0.65$; first six repetitions: $t_{(34)}=4.03, p=$ $3.37 \times 10^{-4}, \beta=0.56$, sr $=0.56$; Table 5). Indeed, looking at the semipartial correlations, it accounted for more variance in alERC volume than MoCA, age, or both combined.

These results suggest that our task may be tapping into a form of cognitive decline that is more sensitive to neurodegenerative changes in alERC volume than the cognitive processes assessed by the MoCA or predicted by chronological age. We note that, with our current sample of participants, age was not a significant predictor for alERC volume even if MoCA was excluded as a predictor. However, this is likely due to our participant selection process (in which the group of participants who scored above the MoCA threshold score were matched in age to the group who scored below the MoCA threshold score), rather than suggesting that age has no effect on alERC volume.

As a post hoc analysis, we looked at whether there were laterality effects in the alERC that affected task performance. We re- 
Table 5. Multiple regression analyses

\begin{tabular}{|c|c|c|c|c|c|c|c|c|c|c|c|c|}
\hline \multirow[b]{2}{*}{ Predictors } & \multicolumn{3}{|l|}{ Repeated } & \multicolumn{3}{|l|}{ Recombined } & \multicolumn{3}{|l|}{ Novel } & \multicolumn{3}{|c|}{ First six repetitions } \\
\hline & $\beta$ & $t$ & sr & $\beta$ & $t$ & $\mathrm{sr}$ & $\beta$ & $t$ & $\mathrm{sr}$ & $\beta$ & $t$ & $\mathrm{sr}$ \\
\hline \multicolumn{13}{|c|}{ Proportion of fixations to middle ROI predicted by regional volume } \\
\hline CA1 & -0.233 & -0.982 & -0.145 & -0.055 & -0.240 & -0.034 & -0.056 & -0.246 & -0.035 & -0.410 & -1.771 & -0.256 \\
\hline Subiculum & -0.080 & -0.339 & -0.050 & -0.060 & -0.264 & -0.037 & 0.192 & 0.842 & 0.121 & -0.151 & -0.655 & -0.095 \\
\hline $\mathrm{DG} / \mathrm{CA} 23$ & -0.123 & -0.564 & -0.083 & -0.176 & -0.842 & -0.120 & -0.022 & -0.104 & -0.015 & 0.066 & 0.311 & 0.045 \\
\hline PRC & -0.301 & -1.441 & -0.213 & -0.198 & -0.986 & -0.140 & -0.219 & -1.083 & -0.155 & -0.201 & -0.983 & -0.142 \\
\hline alERC & $0.603^{* *}$ & 3.603 & 0.534 & $0.663^{* *}$ & 4.127 & 0.586 & $0.733^{* *}$ & 4.512 & 0.648 & $0.652^{* *}$ & 3.987 & 0.577 \\
\hline pmERC & 0.162 & 0.731 & 0.108 & 0.110 & 0.517 & 0.073 & -0.091 & -0.425 & -0.061 & 0.257 & 1.188 & 0.172 \\
\hline \multirow[t]{3}{*}{ PHC } & 0.184 & 1.110 & 0.164 & 0.215 & 1.354 & 0.192 & -0.066 & -0.408 & -0.059 & 0.112 & 0.692 & 0.100 \\
\hline & \multicolumn{3}{|c|}{$F_{(7,27)}=2.66, p=0.03$} & \multicolumn{3}{|c|}{$F_{(7,27)}=3.22, p=0.01$} & \multicolumn{3}{|c|}{$F_{(7,27)}=3.07, p=0.02$} & \multicolumn{3}{|c|}{$F_{(7,27)}=2.96, p=0.02$} \\
\hline & \multicolumn{3}{|c|}{$R^{2}=0.41, R_{\mathrm{adj}}^{2}=0.26$} & \multicolumn{3}{|c|}{$R^{2}=0.46, R_{\mathrm{adj}}^{2}=0.32$} & \multicolumn{3}{|c|}{$R^{2}=0.44, R_{\mathrm{adj}}^{2}=0.30$} & \multicolumn{3}{|c|}{$R^{2}=0.43, R_{\mathrm{adj}}^{2}=0.29$} \\
\hline \multicolumn{13}{|c|}{ Normalized number of fixations to entire object predicted by regional volume } \\
\hline CA1 & -0.100 & -0.362 & -0.062 & 0.118 & 0.408 & 0.074 & -0.025 & -0.090 & -0.016 & -0.294 & -1.107 & -0.184 \\
\hline Subiculum & 0.090 & 0.327 & 0.056 & 0.161 & 0.556 & 0.101 & 0.356 & 1.268 & 0.224 & 0.232 & 0.880 & 0.146 \\
\hline $\mathrm{DG} / \mathrm{CA} 23$ & -0.158 & -0.622 & -0.107 & -0.079 & -0.294 & -0.053 & -0.267 & -1.025 & -0.181 & -0.017 & -0.070 & -0.012 \\
\hline PRC & 0.360 & 1.479 & 0.255 & 0.168 & 0.658 & 0.119 & 0.181 & 0.727 & 0.128 & 0.420 & 1.797 & 0.298 \\
\hline alERC & -0.349 & -1.792 & -0.309 & -0.272 & -1.325 & -0.240 & 0.150 & 0.752 & 0.133 & -0.260 & -1.386 & -0.230 \\
\hline pmERC & -0.191 & -0.742 & -0.128 & -0.277 & -1.020 & -0.185 & -0.434 & -1.644 & -0.290 & -0.352 & -1.421 & -0.236 \\
\hline \multirow[t]{3}{*}{ PHC } & 0.096 & 0.498 & 0.086 & -0.021 & -0.105 & -0.019 & -0.044 & -0.225 & -0.040 & 0.093 & 0.503 & 0.083 \\
\hline & \multicolumn{3}{|c|}{$F_{(7,27)}=0.95, p=0.49$} & \multicolumn{3}{|c|}{$F_{(7,27)}=0.48, p=0.84$} & \multicolumn{3}{|c|}{$F_{(7,27)}=0.72, p=0.66$} & \multicolumn{3}{|c|}{$F_{(7,27)}=1.34, p=0.27$} \\
\hline & \multicolumn{3}{|c|}{$R^{2}=0.20, R_{\mathrm{adj}}^{2}=-0.10$} & \multicolumn{3}{|c|}{$R^{2}=0.11, R_{\text {adj }}^{2}=-0.12$} & \multicolumn{3}{|c|}{$R^{2}=0.16, R_{\mathrm{adj}}^{2}=-0.06$} & \multicolumn{3}{|c|}{$R^{2}=0.26, R_{\mathrm{adj}}^{2}=0.07$} \\
\hline \multicolumn{13}{|c|}{ alERC volume predicted by MoCA, age, and proportion of fixations to middle ROI } \\
\hline MoCA & $0.375^{*}$ & 2.500 & 0.368 & $0.322^{*}$ & 2.369 & 0.320 & $0.429^{* *}$ & 3.362 & 0.418 & $0.364^{*}$ & 2.593 & 0.359 \\
\hline Age & -0.040 & -0.271 & -0.040 & 0.025 & 0.179 & 0.024 & 0.074 & 0.584 & 0.073 & -0.073 & -0.526 & -0.073 \\
\hline \multirow[t]{3}{*}{ Mid-ROl viewing } & $0.490^{* *}$ & 3.291 & 0.484 & $0.588^{* *}$ & 4.323 & 0.584 & $0.674^{* *}$ & 5.253 & 0.653 & $0.563^{* *}$ & 4.029 & 0.558 \\
\hline & \multicolumn{3}{|c|}{$F_{(3,31)}=5.06, p=0.006$} & \multicolumn{3}{|c|}{$F_{(3,31)}=7.95, p<0.001$} & $F_{(3,31}$ & $11.23, p<$ & & $F_{(3,3}$ & $7.05, p=$ & \\
\hline & & $33, R_{\mathrm{adj}}^{2}=$ & & & $44, R_{\text {adj }}^{2}=$ & & & $52, R_{\mathrm{adj}}^{2}=$ & & & $41, R_{\mathrm{adj}}^{2}=$ & \\
\hline
\end{tabular}

Multiple regression models were run separately for trials in each novelty condition (Figure 2 ) and also for trials in the first six repetitions. Each model is shown in its own column. The top section shows multiple regression analyses with brain region volumes as predictors for the proportion of fixations directed to the middle ROl in each novelty condition. Across all conditions, alERC volume was the only significant predictor for fixations to the middle ROI. The middle section shows multiple regression analyses with brain region volumes as predictors for the normalized number of fixations to the entire object in each novelty condition. No brain region was a significant predictor for this variable in any condition. The bottom section shows multiple regression analyses with $\mathrm{MoCA}$, age, and viewing to middle ROl in each novelty condition as predictors for alERC volume. In all conditions, viewing to the middle ROI was a significant predictor for alERC volume, even after accounting for MoCA and age. ${ }^{*} p<0.05,{ }^{* *} p<0.01$.

peated the multiple regression models predicting the proportion of fixations to the middle ROI using brain regions as predictors (as in Table 5), splitting the alERC predictor by hemisphere. This did not show any clear laterality effects: comparing the standardized coefficients (betas) of those models, some conditions revealed a stronger association with the left alERC and other conditions with the right alERC [repeated: 0.332 (left), 0.416 (right); recombined: 0.476 (left), 0.325 (right); novel: 0.454 (left), 0.445 (right); first six repetitions: 0.331 (left), 0.483 (right)].

\section{Discussion}

In this study, we examined older adults' viewing patterns to conjunctive objects and compared them with the volumes of their MTL regions and hippocampus subfields. Crucially, we observed that the proportion of fixations directed to the critical region of a conjunctive object (a measure of configural processing) was significantly and selectively predicted by alERC volume regardless of an object's novelty. This effect was not driven by any changes in overall viewing. Moreover, we showed that individual differences in viewing, reflecting different degrees of configural processing, were related to individual differences in alERC volume, even after accounting for the effects of MoCA score and age. Together, our results demonstrate that alERC volume was strongly associated with attention to the spatial arrangement of the parts of an object. To the best of our knowledge, this is the first study to demonstrate such a role for the alERC and the first to measure the effects of alERC structural volume on cognition.

Previous neuroimaging studies of the human LEC focused on how that region supports object representations (Schultz et al., 2012; Reagh and Yassa, 2014), whereas rodent studies suggested that the putatively homologous LEC represents spatial properties of objects (Deshmukh and Knierim, 2011; Deshmukh et al., 2012; Tsao et al., 2013). This study connects these lines of evidence, suggesting that the human alERC may support intra-item configural processing (i.e., attention to the spatial arrangement of an object's features). In rodents, the LEC has recurrent connections with the MEC (van Strien et al., 2009), which plays an important role in spatial representation (Moser et al., 2014; Sasaki et al., 2015), and with the hippocampus, which supports relational binding (Olsen et al., 2012) and spatial memory (Schiller et al., 2015). There is evidence supporting a similar pattern of functional connectivity in humans (Maass et al., 2015). When combined with the alERC's putative inputs from the perirhinal cortex, which is believed to support object processing (Cowell et al., 2010; Saksida and Bussey, 2010; Ranganath and Ritchey, 2012), this suggests that the alERC is a critical region where information from both the object and spatial pathways converge.

Although item memory has been connected to the alERC using fMRI (Schultz et al., 2012; Reagh and Yassa, 2014) and to the PRC in convergent work across many different methodologies (e.g., Meunier et al., 1993; Henson et al., 2003; Bowles et al., 2007), we did not observe any memory effects in either region. In addition, unlike previous findings from our group (Barense et al., 2012; Peterson et al., 2012; Erez et al., 2016), we did not observe any conjunctive processing effects related to PRC. However, these results are not necessarily contradictory. Because we used eye-tracking/volumetry in older adults rather than fMRI in younger adults or neuropsychological investigations of patients with large PRC/alERC lesions, it is conceivable that these divergent methodologies are differentially sensitive to subtle degrees of damage. For example, it is possible that mnemonic/perceptual 

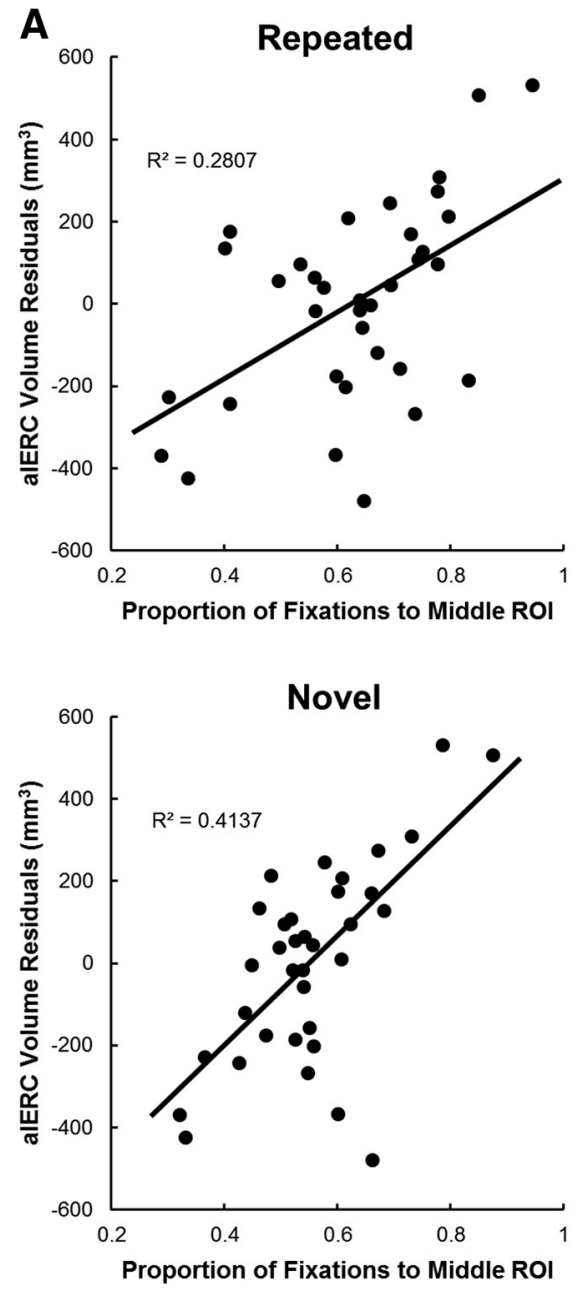
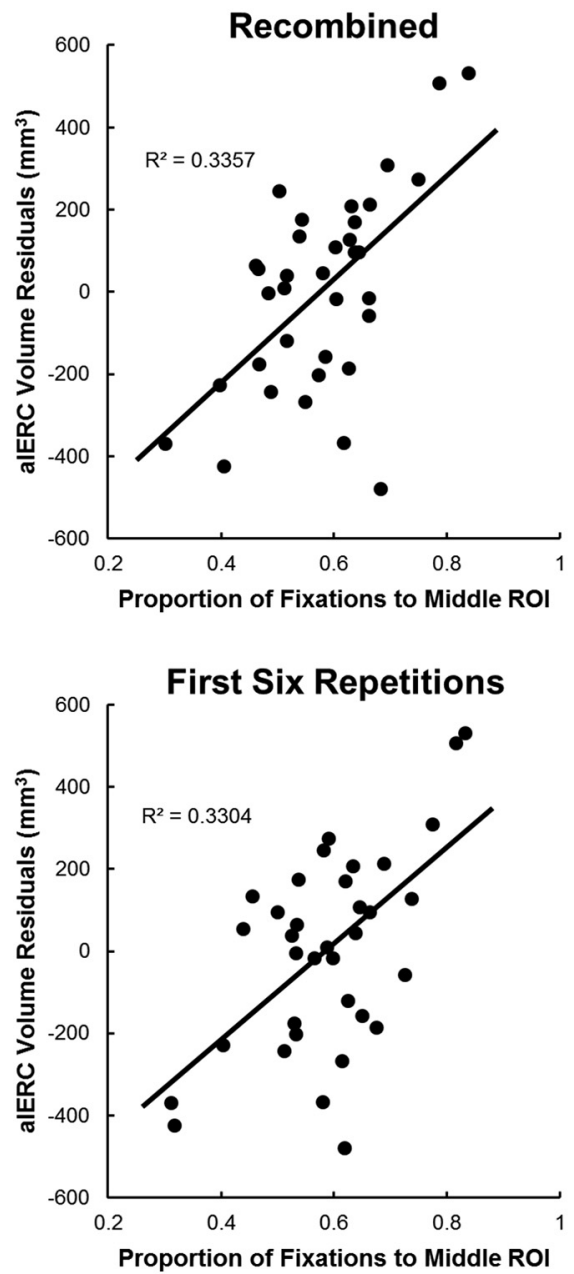

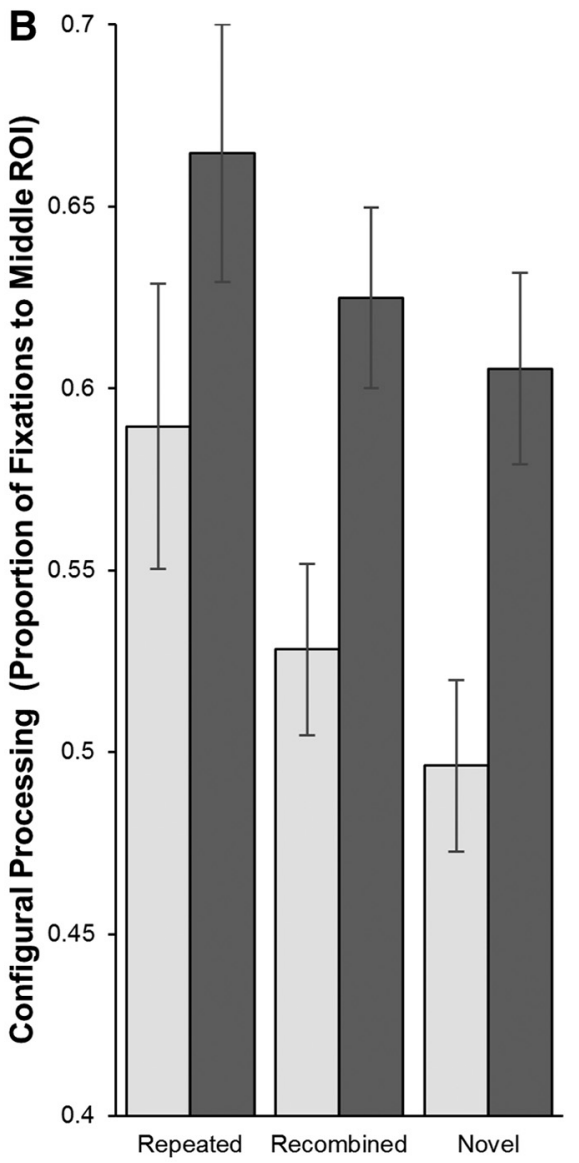

口Smaller alERC $\square$ Larger alERC

Figure 5. A, Correlation plots of the proportion of fixations to middle ROI versus alERC residuals. These depict solely the contribution of the alERC predictor in Table 5 (i.e., with the contribution of other regions removed) and show a strong correlation of alERC structural volume with eye movements to the configurally relevant portion of an object. $B$, Proportion of fixations to middle ROI shown with a median split based on alERC volume demonstrating the same relationship as in the correlation plots. Smaller alERC volumes were associated with reduced viewing of the middle ROI across all novelty conditions. Error bars indicate SEM.

processes related to PRC/alERC-based object representations are more robust and require significant volume (or white matter tract) loss before clear deficits are present, whereas the volume differences here fall below the threshold needed to observe such deficits. However, our results are consistent with findings showing that, when participants with broad MTL lesions (including the alERC) misidentified impossible 2D objects, they showed less viewing to the critical region that identified the object as configurally impossible (Lee and Rudebeck, 2010).

Although we found that age was not a significant predictor for alERC volume after having accounted for the effect of intra-item configural processing, longitudinal structural imaging studies have shown that the volume of MTL regions, including the ERC, decline with age, particularly in older adults over the age of 50 (Raz et al., 2004). Despite our efforts to match participants demographically who scored above and below the MoCA threshold score (and thus minimize the effect of age on regional volumes), it is possible that variation in alERC volume in our sample was related to age. However, this was also likely to be true for all of the other MTL regions we assessed (Raz et al., 2004; La Joie et al., 2013; Wisse et al., 2014). Strikingly, we found that the alERC was the only MTL region with a volume that predicted intra-item configural processing in our experimental task. In this regard, our results are consistent with findings that volume reduction in the entire ERC is associated with poorer memory outcomes in healthy older adults (Rodrigue and Raz, 2004; Fjell et al., 2014).

A particular concern in comparing across different studies of the alERC is the variable definition of its boundaries. We defined the alERC based on histology laterally (Insausti et al., 1998) and on functional connectivity analysis medially (Maass et al., 2015). Our definition of the alERC includes part of the parahippocampal gyrus (adjacent to pmERC) and part of the medial bank of the collateral sulcus (adjacent to PRC). Both of the previous fMRI studies (Schultz et al., 2012; Reagh and Yassa, 2014) that found alERC involvement in object memory placed the alERC mostly on the medial bank of the collateral sulcus. Another group identified a largely coterminous region on the medial bank of the collateral sulcus as the "medial perirhinal cortex" and reported that it plays a role in disambiguating visually similar objects (Kivisaari et al., 2012) and experiences cortical thinning in preclinical Alzheimer's disease (Krumm et al., 2016). The greater medial extent of our alERC might explain the additional role in configural processing of objects that we report. This suggests a continuum in the ERC between more spatial representations in the pmERC and more object-like representations in the alERC. Indeed, cells responsive to spatial properties, cells that are object 
specific, and cells responsive to combinations of spatial and object-specific dimensions are not strictly separated in the two halves of the ERC; rather, they are widely distributed over both regions (Keene et al., 2016). Further work is necessary to pinpoint definitively the functional heterogeneity of the ERC and the corresponding spatial distribution of these functional subregions.

On a more practical level, an eye-tracking-based cognitive task tracking alERC decline might serve as a cost-effective early screening method for Alzheimer's disease pathology (Crutcher et al., 2009) years before traditional cognitive symptoms appear. Both postmortem histology (Braak and Braak, 1991) and in vivo cerebral blood flow imaging (Khan et al., 2014) show that Alzheimer's disease pathology appears earliest in the alERC. Similarly, morphometric analysis shows that the earliest preclinical Alzheimer's disease atrophy can also be found in the alERC (Miller et al., 2015), suggesting that alERC volume may be a sensitive biomarker for preclinical Alzheimer's disease. If our task taps into a form of cognitive change that is particularly sensitive to changes in alERC volume, then it may be useful for identifying asymptomatic preclinical Alzheimer's disease patients for clinical trials, as well as screening for potential Alzheimer's disease when a treatment is developed.

\section{References}

Althoff RR, Cohen NJ (1999) Eye-movement-based memory effect: a reprocessing effect in face perception. J Exp Psychol Learn Mem Cogn 25:9971010. CrossRef Medline

Amaral DG, Insausti R (1990) The hippocampal formation. In: The human nervous system (Paxinos G, ed). San Diego: Academic.

Barense MD, Groen II, Lee AC, Yeung LK, Brady SM, Gregori M, Kapur N, Bussey TJ, Saksida LM, Henson RN (2012) Intact memory for irrelevant information impairs perception in amnesia. Neuron 75:157-167. CrossRef Medline

Bowles B, Crupi C, Mirsattari SM, Pigott SE, Parrent AG, Pruessner JC, Yonelinas AP, Köhler S (2007) Impaired familiarity with preserved recollection after anterior temporal-lobe resection that spares the hippocampus. Proc Natl Acad Sci U S A 104:16382-16387. CrossRef Medline

Braak H, Braak E (1991) Neuropathological stageing of Alzheimer-related changes. Acta Neuropathol 82:239-259. CrossRef Medline

Braak H, BraakE (1992) The human entorhinal cortex: normal morphology and lamina-specific pathology in various diseases. Neurosci Res 15:6-31. CrossRef Medline

Buckner RL, Head D, Parker J, Fotenos AF, Marcus D, Morris JC, Snyder AZ (2004) A unified approach for morphometric and functional data analysis in young, old, and demented adults using automated atlas-based head size normalization: Reliability and validation against manual measurement of total intracranial volume. Neuroimage 23:724-738. CrossRef Medline

Burwell RD (2000) The parahippocampal region: corticocortical connectivity. Ann N Y Acad Sci 911:25-42. Medline

Cowell RA, Bussey TJ, Saksida LM (2010) Components of recognition memory: dissociable cognitive processes or just differences in representational complexity? Hippocampus 20:1245-1262. CrossRef Medline

Crutcher MD, Calhoun-Haney R, Manzanares CM, Lah JJ, Levey AI, Zola SM (2009) Eye tracking during a visual paired comparison task as a predictor of early dementia. Am J Alzheimers Dis Other Demen 24:258-266. CrossRef Medline

Deshmukh SS, Knierim JJ (2011) Representation of non-spatial and spatial information in the lateral entorhinal cortex. Front Behav Neurosci 5:69. CrossRef Medline

Deshmukh SS, Johnson JL, Knierim JJ (2012) Perirhinal cortex represents nonspatial, but not spatial, information in rats foraging in the presence of objects: Comparison with lateral entorhinal cortex. Hippocampus 22: 2045-2058. CrossRef Medline

Dice L (1945) Measures of the amount of ecologic association between species. Ecology 26:297-302. CrossRef

Erez J, Cusack R, Kendall W, Barense MD (2016) Conjunctive coding of complex object features. Cereb Cortex 26:2271-2282. CrossRef Medline

Fjell AM, Westlye LT, Grydeland H, Amlien I, Espeseth T, Reinvang I, Raz N,
Dale AM, Walhovd KB; Alzheimer Disease Neuroimaging Initiative (2014) Accelerating cortical thinning: Unique to dementia or universal in aging? Cereb Cortex 24:919-934. CrossRef Medline

Henson RN, Cansino S, Herron JE, Robb WG, Rugg MD (2003) A familiarity signal in human anterior medial temporal cortex? Hippocampus 13: 301-304. CrossRef Medline

Insausti R, Juottonen K, Soininen H, Insausti AM, Partanen K, Vainio P, Laakso MP, Pitkänen A (1998) MR volumetric analysis of the human entorhinal, perirhinal, and temporopolar cortices. Am J Neuroradiol 19: 659-671. Medline

Jack CR Jr, Petersen RC, Xu YC, Waring SC, O’Brien PC, Tangalos EG, Smith GE, Ivnik RJ, Kokmen E (1997) Medial temporal atrophy on MRI in normal aging and very mild Alzheimer's disease. Neurology 49:786-794. CrossRef Medline

Keene CS, Bladon J, McKenzie S, Liu CD, O’Keefe J, Eichenbaum H (2016) Complementary functional organization of neuronal activity patterns in the perirhinal, lateral entorhinal, and medial entorhinal cortices. J Neurosci 36:3660-3675. CrossRef Medline

Khan UA, Liu L, Provenzano FA, Berman DE, Profaci CP, Sloan R, Mayeux R, Duff KE, Small SA (2014) Molecular drivers and cortical spread of lateral entorhinal cortex dysfunction in preclinical Alzheimer's disease. Nat Neurosci 17:304-311. CrossRef Medline

Kivisaari SL, Tyler LK, Monsch AU, Taylor KI (2012) Medial perirhinal cortex disambiguates confusable objects. Brain 135:3757-3769. CrossRef Medline

Knierim JJ, Neunuebel JP, Deshmukh SS (2014) Functional correlates of the lateral and medial entorhinal cortex: objects, path integration and localglobal reference frames. Philos Trans R Soc Lond B Biol Sci 369:20130369. CrossRef Medline

Krumm S, Kivisaari SL, Probst A, Monsch AU, Reinhardt J, Ulmer S, Stippich C, Kressig RW, Taylor KI (2016) Cortical thinning of parahippocampal subregions in very early Alzheimer's disease. Neurobiol Aging 38:188196. CrossRef Medline

La Joie R, Perrotin A, de La Sayette V, Egret S, Doeuvre L, Belliard S, Eustache F, Desgranges B, Chételat G (2013) Hippocampal subfield volumetry in mild cognitive impairment, Alzheimer's disease and semantic dementia. Neuroimage Clin 3:155-162. CrossRef Medline

Lee AC, Rudebeck SR (2010) Human medial temporal lobe damage can disrupt the perception of single objects. J Neurosci 30:6588-6594. CrossRef Medline

Maass A, Berron D, Libby LA, Ranganath C, Düzel E (2015) Functional subregions of the human entorhinal cortex. Elife 4. CrossRef Medline

Meunier M, Bachevalier J, Mishkin M, Murray EA (1993) Effects on visual recognition of combined and separate ablations of the entorhinal and perirhinal cortex in rhesus monkeys. J Neurosci 13:5418-5432. Medline

Miller MI, Ratnanather JT, Tward DJ, Brown T, Lee DS, Ketcha M, Mori K, Wang MC, Mori S, Albert MS, Younes L (2015) Network neurodegeneration in Alzheimer's Disease via MRI based shape diffeomorphometry and high-field atlasing. Front Bioeng Biotechnol 3:54. CrossRef Medline

Moser EI, Roudi Y, Witter MP, Kentros C, Bonhoeffer T, Moser MB (2014) Grid cells and cortical representation. Nat Rev Neurosci 15:466-481. CrossRef Medline

Naber PA, Caballero-Bleda M, Jorritsma-Byham B, Witter MP (1997) Parallel input to the hippocampal memory system through peri- and postrhinal cortices. Neuroreport 8:2617-2621. CrossRef Medline

Nasreddine ZS, Phillips NA, Bédirian V, Charbonneau S, Whitehead V, Collin I, Cummings JL, Chertkow H (2005) The Montreal Cognitive Assessment, MoCA: a brief screening tool for mild cognitive impairment. J Am Geriatr Soc 53:695-699. CrossRef Medline

Navarro Schröder T, Haak KV, Zaragoza Jimenez NI, Beckmann CF, Doeller CF (2015) Functional topography of the human entorhinal cortex. Elife 4. CrossRef Medline

Norman KA, O'Reilly RC (2003) Modeling hippocampal and neocortical contributions to recognition memory: a complementary-learning-systems approach. Psychol Rev 110:611-646. CrossRef Medline

Olsen RK, Moses SN, Riggs L, Ryan JD (2012) The hippocampus supports multiple cognitive processes through relational binding and comparison. Front Hum Neurosci 6:146. CrossRef Medline

Olsen RK, Palombo DJ, Rabin JS, Levine B, Ryan JD, Rosenbaum RS (2013) Volumetric analysis of medial temporal lobe subregions in developmental amnesia using high-resolution magnetic resonance imaging. Hippocampus 23:855-860. CrossRef Medline 
Olsen RK, Sebanayagam V, Lee Y, Moscovitch M, Grady CL, Rosenbaum RS, Ryan JD (2016) The relationship between eye movements and subsequent recognition: evidence from individual differences and amnesia. Cortex 85:182-193. CrossRef Medline

Olsen RK, Yeung L-K, Noly-Gandon A, D'Angelo MC, Kacollja A, Smith VM, Ryan JD, Barense MD (2017) Human anterolateral entorhinal cortex volumes are associated with cognitive decline in aging prior to clinical diagnosis. Neurobio Aging, in press.

Osterreith PA (1944) Le test de copie d'une figure complexe. Arch Psychol (Geneve) 30:205-220.

Palombo DJ, Amaral RS, Olsen RK, Müller DJ, Todd RM, Anderson AK, Levine B (2013) KIBRA polymorphism is associated with individual differences in hippocampal subregions: evidence from anatomical segmentation using high-resolution MRI. J Neurosci 33:13088-13093. CrossRef Medline

Peterson MA, Cacciamani L, Barense MD, Scalf PE (2012) The perirhinal cortex modulates $\mathrm{V} 2$ activity in response to the agreement between part familiarity and configuration familiarity. Hippocampus 22:1965-1977. CrossRef Medline

Pruessner JC, Köhler S, Crane J, Pruessner M, Lord C, Byrne A, Kabani N, Collins DL, Evans AC (2002) Volumetry of temporopolar, perirhinal, entorhinal and parahippocampal cortex from high-resolution MR images: considering the variability of the collateral sulcus. Cereb Cortex 12:1342-1353. CrossRef Medline

Ranganath C, Ritchey M (2012) Two cortical systems for memory-guided behaviour. Nat Rev Neurosci 13:713-726. CrossRef Medline

Raz N, Rodrigue KM, Head D, Kennedy KM, Acker JD (2004) Differential aging of the medial temporal lobe: a study of a five-year change. Neurology 62:433-438. CrossRef Medline

Reagh ZM, Yassa MA (2014) Object and spatial mnemonic interference differentially engage lateral and medial entorhinal cortex in humans. Proc Natl Acad Sci U S A 111:E4264-E4273. CrossRef Medline

Reitan RM, Wolfson D (1985) The Halstead-Reitan Neuropsychological Test Battery: therapy and clinical interpretation. Tucson, AZ: Neuropsychological.

Ritchey M, Libby LA, Ranganath C (2015) Cortico-hippocampal systems involved in memory and cognition: the PMAT framework, Ed 1. New York: Elsevier.

Rodrigue KM, Raz N (2004) Shrinkage of the entorhinal cortex over five years predicts memory performance in healthy adults. J Neurosci 24:956963. CrossRef Medline

Rolls ET (2016) Pattern separation, completion, and categorisation in the hippocampus and neocortex. Neurobiol Learn Mem 129:4-28. CrossRef Medline

Saksida LM, Bussey TJ (2010) The representational-hierarchical view of amnesia: translation from animal to human. Neuropsychologia 48: 2370-2384. CrossRef Medline

Sasaki T, Leutgeb S, Leutgeb JK (2015) Spatial and memory circuits in the medial entorhinal cortex. Curr Opin Neurobiol 32:16-23. CrossRef Medline
Schiller D, Eichenbaum H, Buffalo EA, Davachi L, Foster DJ, Leutgeb S, Ranganath C (2015) Memory and space: towards an understanding of the cognitive map. J Neurosci 35:13904-13911. CrossRef Medline

Schultz H, Sommer T, Peters J (2012) Direct evidence for domain-sensitive functional subregions in human entorhinal cortex. J Neurosci 32:47164723. CrossRef Medline

Shrout PE, Fleiss JL (1979) Intraclass correlations: uses in assessing rater reliability. Psychol Bull 86:420-428. CrossRef Medline

Suzuki WA, Amaral DG (1994) Topographic organization of the reciprocal connections between the monkey entorhinal cortex and the perirhinal and parahippocampal cortices. J Neurosci 14:1856-1877. Medline

Tsao A, Moser MB, Moser EI (2013) Traces of experience in the lateral entorhinal cortex. Curr Biol 23:399-405. CrossRef Medline

van Strien NM, Cappaert NL, Witter MP (2009) The anatomy of memory: an interactive overview of the parahippocampal-hippocampal network. Nat Rev Neurosci 10:272-282. CrossRef Medline

Warrington EK, James M (1991) Visual Object and Space Perception Battery (VOSP). Oxford: Harcourt Assessment.

Wechsler D (1999) Wechsler Abbreviated Scale of Intelligence. San Antonio, TX: Pearson.

Wechsler D (2008) Wechsler Adult Intelligence Scale, Ed 4. San Antonio, TX: Pearson.

Wechsler D (2009) Wechsler Memory Scale, Ed 4. San Antonio, TX: Pearson.

Wisse LE, Gerritsen L, Zwanenburg JJ, Kuijf HJ, Luijten PR, Biessels GJ, Geerlings MI (2012) Subfields of the hippocampal formation at 7 T MRI: in vivo volumetric assessment. Neuroimage 61:1043-1049. CrossRef Medline

Wisse LE, Biessels GJ, Heringa SM, Kuijf HJ, Koek DH, Luijten PR, Geerlings MI (2014) Hippocampal subfield volumes at 7T in early Alzheimer's disease and normal aging. Neurobiol Aging 35:2039-2045. CrossRef Medline

Witter MP (1993) Organization of the entorhinal-hippocampal system: a review of current anatomical data. Hippocampus 3:33-44. Medline

Wolk DA, Das SR, Mueller SG, Weiner MW, Yushkevich PA; Alzheimer's Disease Neuroimaging Initiative (2017) Medial temporal lobe subregional morphometry using high resolution MRI in Alzheimer's disease. Neurobiol Aging 49:204-213. CrossRef Medline

Yeung L, Ryan JD, Cowell RA, Barense MD (2013) Recognition memory impairments caused by false recognition of novel objects. J Exp Psychol Gen 142:1384-1397. CrossRef Medline

Yushkevich PA, et al. (2015a) Quantitative comparison of 21 protocols for labeling hippocampal subfields and parahippocampal subregions in in vivo MRI: towards a harmonized segmentation protocol. Neuroimage 111:526-541. CrossRef Medline

Yushkevich PA, Pluta JB, Wang H, Xie L, Ding SL, Gertje EC, Mancuso L, Kliot D, Das SR, Wolk DA (2015b) Automated volumetry and regional thickness analysis of hippocampal subfields and medial temporal cortical structures in mild cognitive impairment. Hum Brain Mapp 36:258-287. CrossRef Medline 